\title{
Elasticity Solution of Composite Material Wedge Loaded with a Concentrated Moment
}

\author{
Gang Wang, Purong Jia*, Yongyong Suo, Long Zhang, Leilei Zeng \\ Department of Engineering Mechanics, Northwestern Polytechnical University, Xi'an, China \\ Email: *prjia@nwpu.edu.cn
}

How to cite this paper: Wang, G., Jia, P.R., Suo, Y.Y., Zhang, L. and Zeng, L.L. (2019) Elasticity Solution of Composite Material Wedge Loaded with a Concentrated Moment. Journal of Materials Science and Chemical Engineering, 7, 77-85. https://doi.org/10.4236/msce.2019.78009

Received: May 27, 2019

Accepted: August 25, 2019

Published: August 28, 2019

\begin{abstract}
The complex variable functions are used and analyzed for the solving the mechanic problem of composite plates. The stress boundary condition for composite material wedge is considered. By constructing new stress function, the mechanic analysis of the composite material wedge subjected to a concentrated moment is conducted. The stress boundary problem is studied and the basic governing equation is solved by using the complex function method. The formulae of the stress fields are derived for the wedge loaded with a concentrated moment.
\end{abstract}

\section{Keywords}

Composite Material, Concentrated Moment, Complex Function, Stress Fields

\section{Introduction}

The elasticity analysis of the plane stress problem is of great importance to the usual engineering application. The stress distribution depends on the forces acting on the complete closed boundary. The complex variable theory provides a very powerful tool for the solution of many boundary value problems in the elastic body [1]. Such theory was originally found by some researchers for solving general boundary problems in isotropic materials. Furthermore, the complex variable technique has also been expanded to use for anisotropic materials [2] [3]. Complex variable methods prove to be very useful for the solution of many elastic solid loading problems. Fiber-reinforced polymer matrix materials are the most typical composites, which are also as anisotropic materials at the macroscopic level [4] [5]. The orthotropic plate may have been the base of composites in common engineering use. Typical examples include concentrated force and moment systems applied to the boundary of the plate. The feasible method to 
solve stress-field problems in anisotropic composites is to use the analytic function theory, and the results have been reported [6] [7]. The purpose of this paper is to focus attention on the general solution of the boundary-value problem for the orthotropic materials and to illustrate the method with examples.

\section{Typical Plane Problem and Basic Equations}

Let us now consider a composite wedge subjected to a concentrated moment $M$ at its end as shown in Figure 1. The moment $M$ is in the $x$ - $y$ plane and the thickness of the wedge in the direction perpendicular to the $x$ - $y$ plane is taken as unity, so that $\mathrm{M}$ is the moment per unit thickness. The load distribution along the thickness of the plate is uniform. The conditions along the boundary faces of the wedge are free $(\theta= \pm \alpha)$ and can be satisfied by taking the values to be zero for the stress components, $\sigma_{\theta}, \tau_{r \theta}$. Thus, the boundary conditions are given by:

$$
\sigma_{\theta}=0, \tau_{r \theta}=0 \quad(\text { for } \theta= \pm \alpha)
$$

The plane stress problems of composite materials are common and very importance for the application. It is the key point to solve stress-field problems in orthotropic materials. Suppose the principal elastic directions of the plate coincide with the coordinate directions $(x, y)$, and let the directions 1,2 parallel to the axes $x, y$, respectively.

In the case of plane stress state, the equilibrium equations are as (nobody forces):

$$
\frac{\partial \sigma_{x}}{\partial x}+\frac{\partial \tau_{x y}}{\partial y}=0, \quad \frac{\partial \tau_{x y}}{\partial x}+\frac{\partial \sigma_{y}}{\partial y}=0
$$

Usually, the method of solving the equations is by introducing a typical function $U$ of $x$ and $y$, called the stress function. Through taking any real function $U$, it is easily checked that the equilibrium equations are satisfied by putting the following expressions for the stress components

$$
\sigma_{x}=\frac{\partial^{2} U}{\partial y^{2}}, \sigma_{y}=\frac{\partial^{2} U}{\partial x^{2}}, \tau_{x y}=-\frac{\partial^{2} U}{\partial x \partial y}
$$

By means of original stress and strain relations for orthotropic materials, the governing equation of the compatibility condition can be expressed by the stress function $U(x, y)$, which is

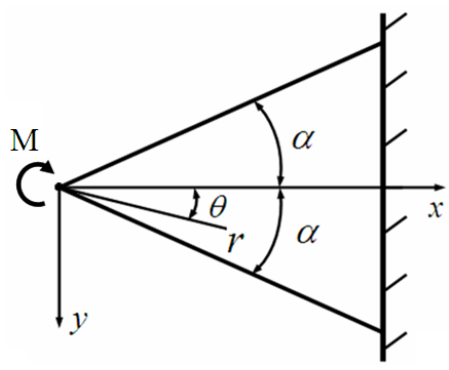

Figure 1. The wedge and boundary condition. 


$$
\frac{\partial^{4} U}{\partial y^{4}}+2\left(\frac{E_{1}}{2 G_{12}}-v_{12}\right) \frac{\partial^{4} U}{\partial x^{2} \partial y^{2}}+\frac{E_{1}}{E_{2}} \frac{\partial^{4} U}{\partial x^{4}}=0
$$

Thus, the solution of a plane problem can be reduce to finding a solution of Equation (4) that must also satisfy the boundary conditions.

\section{Complex Variable and Stress Function}

In order to solve the boundary loading problem about an orthotropic plate, the complex function shall be introduced for the convenience of stress investigation.

\subsection{Complex Variable and Coordinates}

In the solution of the partial differential Equation (4) and also in the construction of suitable stress functions, it is very advantageous to use complex variables. Generally, Two real numbers $x$ and $y$ form the complex number, $z=x+i y$, (with $i^{2}=-1$ ). And the conjugate complex number $\bar{z}=x-i y$ must be used together. For the convenience of general investigation, now another complex variable $(w)$ and its conjugate $(\bar{w})$ are also be introduced and defined by

$$
w=x+i h y=X+i Y, \quad \bar{w}=x-i h y=X-i Y
$$

And so that, $X=x, Y=h y$, where $h$ is a real arbitrary constant. And we suppose the constant $h$ to be positive $(h>0)$, also it can be called tensile or compressive ratio for the coordinate system. The partial derivative relation must be given by

$$
\frac{\partial w}{\partial X}=\frac{\partial w}{\partial x}=\frac{\partial \bar{w}}{\partial X}=\frac{\partial \bar{w}}{\partial x}=1, \frac{\partial w}{\partial Y}=\frac{1}{h} \frac{\partial w}{\partial y}=i, \frac{\partial \bar{w}}{\partial Y}=\frac{1}{h} \frac{\partial \bar{w}}{\partial y}=-i
$$

For the plane problem, rectangular and polar coordinates are shown in Figure 2. In terms of the polar coordinates (in $o x y$-plane and $O X Y$-plane), the complex variables can be written as:

$$
w=r \cos \theta+i h r \sin \theta=R \cos \Theta+i R \sin \Theta
$$

Thus, there are following relations:

$$
\begin{gathered}
x=r \cos \theta, \quad y=r \sin \theta, \quad z \bar{z}=x^{2}+y^{2}=r^{2} \\
R \cos \Theta=r \cos \theta, \quad R \sin \Theta=h r \sin \theta, \quad \tan \Theta=h \tan \theta \\
\tan \beta=h \tan \alpha, \quad w+\bar{w}=2 x, \quad w-\bar{w}=2 i h y \\
w \bar{w}=x^{2}+h^{2} y^{2}=r^{2} \cos ^{2} \theta+h^{2} r^{2} \sin ^{2} \theta=R^{2}\left(\cos ^{2} \Theta+\sin ^{2} \Theta\right)=R^{2}
\end{gathered}
$$
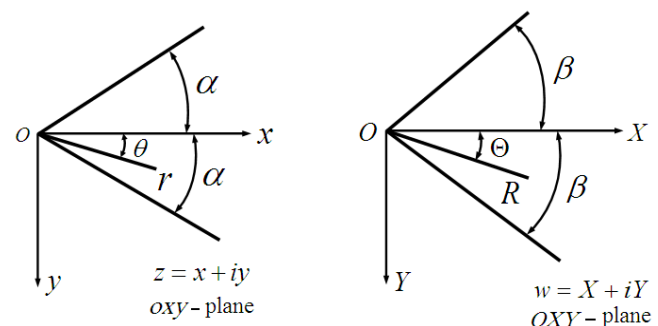

Figure 2. Scheme of the coordinates in the wedge plane. 
When $h=1$, then $\Theta=\theta, R=r$. The w-plane is also reduced to $z$-plane.

On the basis of above definition, the stress function $U$ can be expressed by the complex variables. The partial derivation of $U$ with $x$ or $y$ can be transformed into other expressions with $w(\bar{w})$, namely

$$
\begin{gathered}
\frac{\partial U}{\partial x}=\frac{\partial U}{\partial w} \frac{\partial w}{\partial x}+\frac{\partial U}{\partial \bar{w}} \frac{\partial \bar{w}}{\partial x}=\frac{\partial U}{\partial w}+\frac{\partial U}{\partial \bar{w}} \\
\frac{\partial U}{\partial y}=\frac{\partial U}{\partial w} \frac{\partial w}{\partial y}+\frac{\partial U}{\partial \bar{w}} \frac{\partial \bar{w}}{\partial y}=i h\left(\frac{\partial U}{\partial w}-\frac{\partial U}{\partial \bar{w}}\right)
\end{gathered}
$$

Then we obtain

$$
\left\{\begin{array}{l}
\frac{\partial^{2} U}{\partial x^{2}}=\frac{\partial^{2} U}{\partial w^{2}}+2 \frac{\partial^{2} U}{\partial w \partial \bar{w}}+\frac{\partial^{2} U}{\partial \bar{w}^{2}} \\
\frac{\partial^{2} U}{\partial y^{2}}=-h^{2}\left(\frac{\partial^{2} U}{\partial w^{2}}-2 \frac{\partial^{2} U}{\partial w \partial \bar{w}}+\frac{\partial^{2} U}{\partial \bar{w}^{2}}\right) \\
\frac{\partial^{2} U}{\partial x \partial y}=i h\left(\frac{\partial^{2} U}{\partial w^{2}}-\frac{\partial^{2} U}{\partial \bar{w}^{2}}\right)
\end{array}\right.
$$

By substituting above equations into the governing Equation (4), we obtain the partial derivative equation with complex variables in the following way

$$
\begin{aligned}
& \left(h^{4}-2 B h^{2}+C\right)\left(\frac{\partial^{4} U}{\partial w^{4}}+\frac{\partial^{4} U}{\partial \bar{w}^{4}}\right)+4\left(C-h^{4}\right)\left(\frac{\partial^{2}}{\partial w^{2}}+\frac{\partial^{2}}{\partial \bar{w}^{2}}\right) \frac{\partial^{2} U}{\partial w \partial \bar{w}} \\
& +2\left(3 h^{4}+2 B h^{2}+3 C\right) \frac{\partial^{4} U}{\partial w^{2} \partial \bar{w}^{2}}=0
\end{aligned}
$$

where, $B=\frac{E_{1}}{2 G_{12}}-v_{12}, C=\frac{E_{1}}{E_{2}}$. Next, we shall solve this equation. We may divide it into the following two cases.

Case I: $\frac{\partial^{2} U}{\partial w \partial \bar{w}} \neq 0, \frac{\partial^{4} U}{\partial w^{2} \partial \bar{w}^{2}}=0$.

In this case, the coefficient parts of other terms in Equation (9) must become zero. Thus, two characteristic equations are given as follows

$$
h^{4}-2 B h^{2}+C=0, C-h^{4}=0
$$

Obviously, the solution ought to be that $h^{4}=C, h^{2}=B$. Hence it leads to

$$
h=\sqrt{\frac{E_{1}}{2 G_{12}}-v_{12}}=\sqrt[4]{\frac{E_{1}}{E_{2}}}
$$

Case II: $\frac{\partial^{2} U}{\partial w \partial \bar{w}}=0$.

In this case, the coefficient part of the first terms in Equation (9) must become zero. Thus, the characteristic equation is given as

$$
h^{4}-2 B h^{2}+C=0
$$

Obviously, the solution may be as

$$
h^{2}=B \pm \sqrt{B^{2}-C} \quad\left(\text { for } B^{2}>C\right)
$$

Let $h_{1}>h_{2}>0$. Then we have 


$$
h_{1}=\sqrt{B+\sqrt{B^{2}-C}}, h_{2}=\sqrt{B-\sqrt{B^{2}-C}}
$$

So that

$$
\left\{\begin{array}{l}
h_{1}=\sqrt{\frac{E_{1}}{2 G_{12}}-v_{12}+\sqrt{\left(\frac{E_{1}}{2 G_{12}}-v_{12}\right)^{2}-\frac{E_{1}}{E_{2}}}} \\
h_{2}=\sqrt{\frac{E_{1}}{2 G_{12}}-v_{12}-\sqrt{\left(\frac{E_{1}}{2 G_{12}}-v_{12}\right)^{2}-\frac{E_{1}}{E_{2}}}}
\end{array}\right.
$$

In view of this, there are two complex variables $\left(w_{1}, w_{2}\right)$.

$$
w_{1}=x+i h_{1} y=r\left(\cos \theta+i h_{1} \sin \theta\right), \quad w_{2}=x+i h_{2} y=r\left(\cos \theta+i h_{2} \sin \theta\right)
$$

Up to now, we complete the construction of the stress function $U$ with complex variables. In the following, we shall give an example to show the application.

\subsection{Stress Expression by Complex Function}

The complex function $\ln w$ shall be considered firstly to use for solving the boundary loading problem as shown in Figure 1. In terms of the boundary condition and experimental knowledge, the real stress function $U$ can be determined by different forms.

For Case I, we may take the stress function $U$ as

$$
U=A_{1} i(\ln w-\ln \bar{w})+A_{2} i\left(\frac{\bar{w}}{w}-\frac{w}{\bar{w}}\right)
$$

Thus the first order partial derivatives can be easily obtained as follows

$$
\begin{gathered}
\frac{\partial U}{\partial x}=A_{1} i\left(\frac{1}{w}-\frac{1}{\bar{w}}\right)+A_{2} i\left(\frac{1}{w}-\frac{1}{\bar{w}}+\frac{w}{\bar{w}^{2}}-\frac{\bar{w}}{w^{2}}\right) \\
\frac{\partial U}{\partial y}=-h A_{1}\left(\frac{1}{w}+\frac{1}{\bar{w}}\right)+h A_{2}\left(\frac{1}{w}+\frac{1}{\bar{w}}+\frac{w}{\bar{w}^{2}}+\frac{\bar{w}}{w^{2}}\right)
\end{gathered}
$$

The second order partial derivatives are

$$
\left\{\begin{array}{l}
\frac{\partial^{2} U}{\partial x^{2}}=A_{1} i\left(\frac{1}{\bar{w}^{2}}-\frac{1}{w^{2}}\right)+2 A_{2} i\left(\frac{1}{\bar{w}^{2}}-\frac{1}{w^{2}}+\frac{\bar{w}}{w^{3}}-\frac{w}{\bar{w}^{3}}\right) \\
\frac{\partial^{2} U}{\partial y^{2}}=i h^{2} A_{1}\left(\frac{1}{w^{2}}-\frac{1}{\bar{w}^{2}}\right)+2 i h^{2} A_{2}\left(\frac{1}{\bar{w}^{2}}-\frac{1}{w^{2}}+\frac{w}{\bar{w}^{3}}-\frac{\bar{w}}{w^{3}}\right) \\
\frac{\partial^{2} U}{\partial x \partial y}=h A_{1}\left(\frac{1}{w^{2}}+\frac{1}{\bar{w}^{2}}\right)-2 h A_{2}\left(\frac{\bar{w}}{w^{3}}+\frac{w}{\bar{w}^{3}}\right)
\end{array}\right.
$$

On the basis of above equations, the stresses can be expressed as:

$$
\left\{\begin{array}{l}
\sigma_{x}=\frac{\partial^{2} U}{\partial y^{2}}=4 h^{3} \frac{x y}{w^{2} \bar{w}^{2}}\left(A_{1}-2 A_{2} \frac{3 x^{2}-h^{2} y^{2}}{w \bar{w}}\right) \\
\sigma_{y}=\frac{\partial^{2} U}{\partial x^{2}}=\frac{4 h x y}{w^{2} \bar{w}^{2}}\left(-A_{1}+2 A_{2} \frac{x^{2}-3 h^{2} y^{2}}{w \bar{w}}\right) \\
\tau_{x y}=-\frac{\partial^{2} U}{\partial x \partial y}=\frac{2 h}{w^{2} \bar{w}^{2}}\left[-A_{1}\left(x^{2}-h^{2} y^{2}\right)+2 A_{2}\left(\frac{x^{4}-6 h^{2} x^{2} y^{2}+h^{4} y^{4}}{w \bar{w}}\right)\right]
\end{array}\right.
$$


In order to solve the stress boundary problem of the wedge, we can consider to taking the relations between stresses in the two coordinate systems. It is common knowledge that the transformation of stresses can be expressed by the following relations:

$$
\left\{\begin{array}{l}
\sigma_{r}=\sigma_{x} \cos ^{2} \theta+\sigma_{y} \sin ^{2} \theta+2 \tau_{x y} \sin \theta \cos \theta \\
\sigma_{\theta}=\sigma_{x} \sin ^{2} \theta+\sigma_{y} \cos ^{2} \theta-2 \tau_{x y} \sin \theta \cos \theta \\
\tau_{r \theta}=\left(\sigma_{y}-\sigma_{x}\right) \sin \theta \cos \theta+\tau_{x y}\left(\cos ^{2} \theta-\sin ^{2} \theta\right)
\end{array}\right.
$$

Substituting the stresses of the expression (14) into the above equations, and using two coordinate relations, $x=r \cos \theta, y=r \sin \theta$ to simplify them, then the stress components $\sigma_{r}, \sigma_{\theta}, \tau_{r \theta}$ in the polar coordinate system can be obtained by

$$
\begin{aligned}
& \sigma_{r}=\frac{2 A_{1} h r^{2}}{w^{2} \bar{w}^{2}}\left(h^{2}-1\right) \sin 2 \theta+\frac{4 A_{2} h r^{4}}{w^{3} \bar{w}^{3}}\left[1-3 h^{2}+\left(h^{4}-1\right) \sin ^{2} \theta\right] \sin 2 \theta \\
& \sigma_{\theta}=0, \tau_{r \theta}=\frac{2 h}{w \bar{w}}\left(-A_{1}+2 A_{2} \frac{1-h^{2} \tan ^{2} \theta}{1+h^{2} \tan ^{2} \theta}\right)
\end{aligned}
$$

In order for the stress fields to meet the necessity of the free boundary conditions at $\theta= \pm \alpha$ shown in Figure 1, the shear stress must be zero. That is to say, $\tau_{r \theta}( \pm \alpha)=0$. Thus, we can obtain the relation of the constants $A_{1}$ and $A_{2}$ in the following

$$
A_{1}=2 A_{2} \frac{1-h^{2} \tan ^{2} \alpha}{1+h^{2} \tan ^{2} \alpha}=2 A_{2} \frac{1-\tan ^{2} \beta}{1+\tan ^{2} \beta}=2 A_{2} \cos 2 \beta
$$

where, $\tan \beta=h \tan \alpha$. Besides that, $\sigma_{\theta}=0$, other stress components are given by

$$
\left\{\begin{array}{l}
\sigma_{r}=\frac{4 A_{2} h r^{2}}{w^{2} \bar{w}^{2}}\left[\left(h^{2}-1\right) \cos 2 \beta+\frac{1-3 h^{2}+\left(h^{4}-1\right) \sin ^{2} \theta}{\cos ^{2} \theta+h^{2} \sin ^{2} \theta}\right] \sin 2 \theta \\
\tau_{r \theta}=\frac{4 A_{2} h}{w \bar{w}}\left(\frac{1-h^{2} \tan ^{2} \theta}{1+h^{2} \tan ^{2} \theta}-\cos 2 \beta\right)
\end{array}\right.
$$

Next, the constant $A_{2}$ ought to be determined. In terms of the loading condition and the coordinate systems, the moment must be in equilibrium. So the main equilibrant equation is given as

$$
\int_{-\alpha}^{\alpha} \tau_{r \theta} r^{2} d \theta+M=0
$$

Pay the attention to $w \bar{w}=r^{2}\left(\cos ^{2} \theta+h^{2} \sin ^{2} \theta\right)$. Then the equilibrant equation must be by

$$
\int_{-\alpha}^{\alpha} \frac{4 A_{2} h}{\left(\cos ^{2} \theta+h^{2} \sin ^{2} \theta\right)}\left(\frac{1-h^{2} \tan ^{2} \theta}{1+h^{2} \tan ^{2} \theta}-\cos 2 \beta\right) d \theta+M=0
$$

By solving the integration (note that: $h>0$ ), the solution can be obtained as

$$
\left[\left(\frac{h^{2}}{h^{2}-1}\right) \frac{h \tan \theta}{\cos ^{2} \theta+h^{2} \sin ^{2} \theta}-\frac{h \tan \theta}{h^{2}-1}-\cos 2 \beta \arctan (h \tan \theta)\right]_{-\alpha}^{\alpha}=-\frac{M}{4 A_{2}}
$$

Because that, $\tan \beta=h \tan \alpha$, then $\beta=\arctan (h \tan \alpha)$. And finally, the con- 
stant is defined as

$$
4 A_{2}=\frac{M}{2 \beta \cos 2 \beta-\sin 2 \beta}
$$

Therefore, the stress components can be determined by the expression (17).

For Case II, we may take the stress function $U$ as

$$
U=D_{1} i\left(\ln w_{1}-\ln \bar{w}_{1}\right)+D_{2} i\left(\ln w_{2}-\ln \bar{w}_{2}\right)
$$

where, $w_{1}=x+i h_{1} y, \quad w_{2}=x+i h_{2} y, \quad \bar{w}_{1}=x-i h_{1} y, \quad \bar{w}_{2}=x-i h_{2} y$. Thus the partial derivatives can be easily obtained as follows

$$
\begin{gathered}
\frac{\partial U}{\partial x}=D_{1}\left(\frac{i}{w_{1}}-\frac{i}{\bar{w}_{1}}\right)+D_{2}\left(\frac{i}{w_{2}}-\frac{i}{\bar{w}_{2}}\right) \\
\frac{\partial U}{\partial y}=-D_{1} h_{1}\left(\frac{1}{w_{1}}+\frac{1}{\bar{w}_{1}}\right)-D_{2} h_{2}\left(\frac{1}{w_{2}}+\frac{1}{\bar{w}_{2}}\right) \\
\frac{\partial^{2} U}{\partial x^{2}}=D_{1}\left(\frac{i}{\bar{w}_{1}^{2}}-\frac{i}{w_{1}^{2}}\right)+D_{2}\left(\frac{i}{\bar{w}_{2}^{2}}-\frac{i}{w_{2}^{2}}\right) \\
\frac{\partial^{2} U}{\partial y^{2}}=D_{1} h_{1}^{2}\left(\frac{i}{w_{1}^{2}}-\frac{i}{\bar{w}_{1}^{2}}\right)+D_{2} h_{2}^{2}\left(\frac{i}{w_{2}^{2}}-\frac{i}{\bar{w}_{2}^{2}}\right) \\
\frac{\partial^{2} U}{\partial x \partial y}=D_{1} h_{1}\left(\frac{1}{w_{1}^{2}}+\frac{1}{\bar{w}_{1}^{2}}\right)+D_{2} h_{2}\left(\frac{1}{w_{2}^{2}}+\frac{1}{\bar{w}_{2}^{2}}\right)
\end{gathered}
$$

On the basis of above equations, the stress components can be determined by

$$
\left\{\begin{array}{l}
\sigma_{x}=\frac{\partial^{2} U}{\partial y^{2}}=D_{1} h_{1}^{2}\left(\frac{i}{w_{1}^{2}}-\frac{i}{\bar{w}_{1}^{2}}\right)+D_{2} h_{2}^{2}\left(\frac{i}{w_{2}^{2}}-\frac{i}{\bar{w}_{2}^{2}}\right) \\
\sigma_{y}=\frac{\partial^{2} U}{\partial x^{2}}=D_{1}\left(\frac{i}{\bar{w}_{1}^{2}}-\frac{i}{w_{1}^{2}}\right)+D_{2}\left(\frac{i}{\bar{w}_{2}^{2}}-\frac{i}{w_{2}^{2}}\right) \\
\tau_{x y}=-\frac{\partial^{2} U}{\partial x \partial y}=-D_{1} h_{1}\left(\frac{1}{w_{1}^{2}}+\frac{1}{\bar{w}_{1}^{2}}\right)-D_{2} h_{2}\left(\frac{1}{w_{2}^{2}}+\frac{1}{\bar{w}_{2}^{2}}\right)
\end{array}\right.
$$

Substituting above stresses into Equation (15), and using two coordinate relations, $x=r \cos \theta, y=r \sin \theta$, the complex variables become of $w_{1}=r\left(\cos \theta+i h_{1} \sin \theta\right), \quad w_{2}=r\left(\cos \theta+i h_{2} \sin \theta\right), \quad \bar{w}_{1}=r\left(\cos \theta-i h_{1} \sin \theta\right)$, $\bar{w}_{2}=r\left(\cos \theta-i h_{2} \sin \theta\right)$. And also to simplify some functions, then the stress components $\sigma_{r}, \sigma_{\theta}, \tau_{r \theta}$ in the polar coordinate system can be obtained as

$$
\begin{gathered}
\sigma_{r}=\frac{4 \sin \theta \cos \theta}{r^{2}}\left[\frac{D_{1} h_{1}\left(h_{1}^{2}-1\right)}{\left(\cos ^{2} \theta+h_{1}^{2} \sin ^{2} \theta\right)^{2}}+\frac{D_{2} h_{2}\left(h_{2}^{2}-1\right)}{\left(\cos ^{2} \theta+h_{2}^{2} \sin ^{2} \theta\right)^{2}}\right] \\
\sigma_{\theta}=0, \quad \tau_{r \theta}=-\frac{2}{r^{2}}\left(\frac{D_{1} h_{1}}{\cos ^{2} \theta+h_{1}^{2} \sin ^{2} \theta}+\frac{D_{2} h_{2}}{\cos ^{2} \theta+h_{2}^{2} \sin ^{2} \theta}\right)
\end{gathered}
$$

In order for the stress fields to meet the necessity of the free boundary conditions at $\theta= \pm \alpha$, the shear stress must be zero, $\tau_{r \theta}( \pm \alpha)=0$. Thus the relation of the constants can be obtained by

$$
\frac{D_{1} h_{1}}{\cos ^{2} \alpha+h_{1}^{2} \sin ^{2} \alpha}+\frac{D_{2} h_{2}}{\cos ^{2} \alpha+h_{2}^{2} \sin ^{2} \alpha}=0
$$


This shows that,

$$
\left\{\begin{array}{l}
D_{2} h_{2}=-D_{1} h_{1} \lambda \\
\lambda=\frac{\cos ^{2} \alpha+h_{2}^{2} \sin ^{2} \alpha}{\cos ^{2} \alpha+h_{1}^{2} \sin ^{2} \alpha}=\frac{1+h_{2}^{2} \tan ^{2} \alpha}{1+h_{1}^{2} \tan ^{2} \alpha}
\end{array}\right.
$$

Thus the stress components are given by

$$
\left\{\begin{array}{l}
\sigma_{r}=\frac{4 D_{1} h_{1}}{r^{2}}\left[\frac{\left(h_{1}^{2}-1\right) \sin \theta \cos \theta}{\left(\cos ^{2} \theta+h_{1}^{2} \sin ^{2} \theta\right)^{2}}-\frac{\lambda\left(h_{2}^{2}-1\right) \sin \theta \cos \theta}{\left(\cos ^{2} \theta+h_{2}^{2} \sin ^{2} \theta\right)^{2}}\right] \\
\sigma_{\theta}=0 \\
\tau_{r \theta}=-\frac{2 D_{1} h_{1}}{r^{2}}\left(\frac{1}{\cos ^{2} \theta+h_{1}^{2} \sin ^{2} \theta}-\frac{\lambda}{\cos ^{2} \theta+h_{2}^{2} \sin ^{2} \theta}\right)
\end{array}\right.
$$

Next, the constant $D_{1}$ ought to be determined. The moment must be in equilibrium, and also the main equilibrant equation is given as

$$
\begin{gathered}
\int_{-\alpha}^{\alpha} \tau_{r \theta} r^{2} d \theta+M=0 \\
-2 D_{1} h_{1} \int_{-\alpha}^{\alpha}\left(\frac{1}{\cos ^{2} \theta+h_{1}^{2} \sin ^{2} \theta}-\frac{\lambda}{\cos ^{2} \theta+h_{2}^{2} \sin ^{2} \theta}\right) d \theta+M=0
\end{gathered}
$$

Hence the moment equilibrant equation must be by

$$
4 D_{1} h_{1}\left[\frac{1}{h_{1}} \arctan \left(h_{1} \tan \alpha\right)-\frac{\lambda}{h_{2}} \arctan \left(h_{2} \tan \alpha\right)\right]=M
$$

That is to say,

$$
\left\{\begin{array}{l}
4 D_{1} h_{1}=\frac{M h_{1} h_{2}}{h_{2} \beta_{1}-\lambda h_{1} \beta_{2}} \\
\beta_{1}=\arctan \left(h_{1} \tan \alpha\right) \quad, \quad \beta_{2}=\arctan \left(h_{2} \tan \alpha\right)
\end{array}\right.
$$

Therefore, the stress components can be determined by

$$
\left\{\begin{array}{l}
\sigma_{r}=\frac{M h_{1} h_{2}}{\left(h_{2} \beta_{1}-\lambda h_{1} \beta_{2}\right) r^{2}}\left[\frac{\left(h_{1}^{2}-1\right) \sin \theta \cos \theta}{\left(\cos ^{2} \theta+h_{1}^{2} \sin ^{2} \theta\right)^{2}}-\frac{\lambda\left(h_{2}^{2}-1\right) \sin \theta \cos \theta}{\left(\cos ^{2} \theta+h_{2}^{2} \sin ^{2} \theta\right)^{2}}\right] \\
\sigma_{\theta}=0 \\
\tau_{r \theta}=-\frac{M h_{1} h_{2}}{2\left(h_{2} \beta_{1}-\lambda h_{1} \beta_{2}\right) r^{2}}\left(\frac{1}{\cos ^{2} \theta+h_{1}^{2} \sin ^{2} \theta}-\frac{\lambda}{\cos ^{2} \theta+h_{2}^{2} \sin ^{2} \theta}\right)
\end{array}\right.
$$

Obviously, the expressions of the stress field distribution are also complex. For the isotropic materials ( $h=1$ ), the expressions will be simplified greatly.

\section{Conclusion}

The mechanical analysis of the composite material wedge subjected to a concentrated moment is conducted by constructing new stress function. The stress boundary problem is studied and the basic governing equation is solved by using the complex function method. The stress fields of the wedge loaded with a concentrated moment are derived, and the general expressions can be simplified for isotropic materials. 


\section{Acknowledgements}

The authors are very wishing to acknowledge the financial support of the Natural Science Foundation of China (NSFC Grant No. 51275422, 51475372).

\section{Conflicts of Interest}

The authors declare no conflicts of interest regarding the publication of this paper.

\section{References}

[1] Timoshenko, S.P. and Goodier, J.N. (1970) Theory of Elasticity. 3rd Edition, McGraw Hill Companies, Inc., New York. https://doi.org/10.1115/1.3408648

[2] Sih, G.C. (1991) Mechanics of Fracture Initiation and Propagation. Kluwer Academic Pub. https://doi.org/10.1007/978-94-011-3734-8

[3] Friedrich, K. (1989) Application of Fracture Mechanics to Composite Materials. Elsevier Sci. Pub.

[4] Sadd, M.H. (2014) Elasticity: Theory, Applications, and Numerics. 3rd Edition, Elsevier Inc., Academic Pres.

[5] Fakoor, M. and Khansari, N.M. (2016) Mixed Mode I/II Fracture Criterion for Orthotropic Materials Based on Damage Zone Properties. Eng Fract Mech, 153, 407-420. https://doi.org/10.1016/j.engfracmech.2015.11.018

[6] Fallah, N. and Nikraftar, N. (2018) Meshless Finite Volume Method for the Analysis of Fracture Problems in Orthotropic Media. Eng Fract Mech, 204, 46-62.

https://doi.org/10.1016/j.engfracmech.2018.09.029

[7] Zhang, H. and Qiao, P. (2019) A State-Based Peridynamic Model for Quantitative Elastic and Fracture Analysis of Orthotropic Materials. Eng Fract Mech, 206, 147-171. https://doi.org/10.1016/j.engfracmech.2018.10.003 